\title{
Propuesta de sendero interpretativo ecoturístico - educativo en el área protegida Refugio de Vida Silvestre Río Escalante - Chacocente, Carazo, Nicaragua
}

\section{Proposal for an ecotourism interpretive path - educational in the protected area Refugio de Vida Silvestre Río Escalante - Chacocente, Carazo, Nicaragua}

\author{
Yuri Lisseth Alemán Jirón ${ }^{1}$, Emelina Tapia Lorío², Karla Alguera Oviedo ${ }^{3}$ \\ ${ }^{1}$ Ingeniera en Recursos Naturales Renovables graduada en la Universidad Nacional Agraria, Managua, Nicaragua \\ ${ }^{2}$ MSc. en Manejo y Conservación de los Recursos Naturales: ORCID: https://orcid.org/0000-0002-9837-7935 / emelina.tapia@ci.una.edu.ni \\ ${ }^{3}$ MSc. en Educación Superior: ORCID: https://orcid.org/0000-0002-8960-717X / kalguera@ci.una.edu.ni, \\ Universidad Nacional Agraria - Facultad de Recursos Naturales y del Ambiente
}

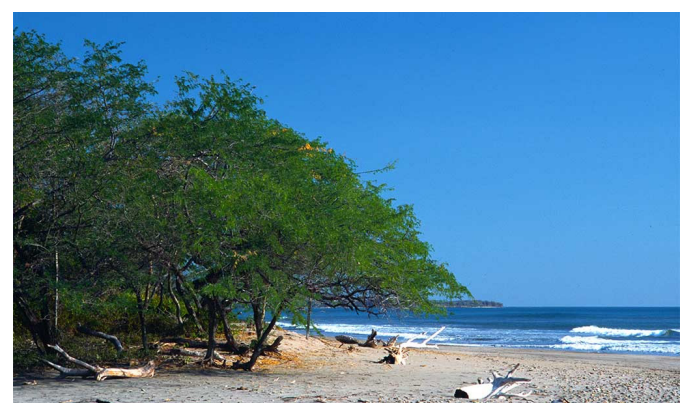

\section{RESUMEN}

El ecoturismo es una modalidad turística ambientalmente responsable que promueve la conservación de los recursos naturales en diversas áreas que cuentan con espacios y destinos con recursos naturales diversos. En este sentido, se puede encontrar este tipo de turismo en espacios naturales como las áreas protegidas en Nicaragua. Este estudio se realizó en el Refugio de Vida Silvestre Río Escalante - Chacocente, ubicado entre el municipio de Santa Teresa, departamento de Carazo y Municipio de Tola, departamento de Rivas, con el objetivo de realizar una propuesta de senderos interpretativos ecoturístico-educativo en esta área protegida. Se contemplaron tres fases metodológicas: (1) organización, revisión de la información, coordinación con las entidades del Ministerio de Recursos Naturales y del Ambiente y planificación de las visitas de reconocimiento; (2) toma de datos de campo, recorridos, observaciones in situ, geo referenciacion de puntos y senderos e identificación de especies de flora arborescente, avifauna silvestre y de potenciales naturales; y (3), análisis y descripción de la información para la elaboración de la propuesta de estaciones (paradas temáticas), del mapa temático, de diseño de rótulos interpretativos y edición del material didáctico (cartilla). Como resultados se elaboró el mapa de dos sendero interpretativo ecoturístico-educativo lineal-circular con nueve estaciones (cinco para el sendero Lagarto Cola Chata y cuatro para el sendero Tamarindo), la propuesta de diseños de cinco rótulos interpretativos; y de una cartilla como material educativo llamada "Haciendo Educación Ambiental", que es dirigida a turistas, comunitarios, investigadores y estudiantes, y contiene información importante para la conservación de la biodiversidad del Refugio de Vida Silvestre Río Escalante - Chacocente y la promoción del turismo sostenible en esta zona de importancia ecológica para el país. Palabras clave: educación ambiental, espacios naturales protegidos, conservación y turismo sostenible.

Recibido: 16 de octubre del 2019

Aceptado: 24 de enero del 2020
ABSTRACT
Ecotourism is an environmentally responsible tourism modality that promotes the conservation of natural resources in various areas that have spaces and destinations with diverse natural resources. In this sense, you can find this type of tourism in natural spaces such as protected areas in Nicaragua. The study was carried out in the Río Escalante - Chacocente Wildlife Refuge, located between the municipality of Santa Teresa, department of Carazo and Tola, department of Rivas, with the aim of making a proposal for interpretive ecotourism-educational trails in this protected area. Three methodological stages were considered: (1) that of organization, review of information, coordination with MARENA entities, and planning of reconnaissance visits; (2) Taking field data, routes, observations in situ, geo-referencing of points and trails, and identification of species of arborescent flora, wild avifauna and natural potentials; and (3), analysis and description of the information collected for the preparation of the station proposal (thematic stops), the thematic map, the design of interpretive signs and editing of the teaching material (booklet). As a result, a map of two linear-circular ecotourism-educational interpretive trails was drawn up with nine stations (five for the Lagarto Cola Chata trail and four for the Tamarindo trail), proposing the designs of five interpretive signs; and a booklet as an educational material called "Doing Environmental Education", which is aimed at tourists, community members, researchers and students, and contains important information for the conservation of the biodiversity of the Río Escalante - Chacocente Wildlife Refuge and the promotion of tourism sustainable in this area of ecological importance for the country.

Keywords: Environmental education, protected natural spaces, conservation and sustainable tourism. 
$\mathbf{N}$ icaragua, como parte de Latinoamérica en los correspondientes procesos de globalización y estabilización socioeconómica, ha empezado a identificar al turismo como una de las principales oportunidades del siglo XXI (Olivares y Obiol, 2005). El turismo en áreas naturales con fines recreativos se ha convertido en la actualidad, en una actividad económica muy importante, puesto que en los últimos años ha venido visualizándose como uno de los sectores más productivos de la economía de muchos países (Ejzman, 2000).

Este creciente aumento de visitación de áreas naturales acarrea como consecuencias, la necesidad de desarrollar herramientas que permitan mejorar el manejo de estas áreas, con rigor en la planificación para alcanzar los objetivos por los cuales fue creada (Cifuentes, Mezquita y Méndez, 1999).

El área protegida Refugio de Vida Silvestre Rio Escalante - Chacocente (RVS), fue creado pensando fundamentalmente en la protección de las tortugas marinas y la conservación del bosque tropical seco, siendo de importancia nacional e internacional; alberga una cantidad de ecosistemas en peligro de extinción como lo indica el Ministerio de Recursos Naturales y del Ambiente (MARENA, 2008).

En este sentido, y siendo un espacio natural protegido, se logró con esta propuesta desarrollar dentro del área, la promoción de sus potenciales naturales a través de senderos interpretativos educativos, integrando lo ecológicos con el ecoturismo y la educación ambiental; con la actualización de la información recabada en los senderos con que cuenta el Refugio, se generaron elementos importantes con información visual al momento de realizar actividades de interés durante los recorridos in situ. Con la elaboración de una cartilla didáctica se brinda información secundaria del área protegida tanto para visitantes nacionales, extranjeros, así como para los pobladores locales; y finalmente, el propósito esperado con el trabajo es ayudar a enriquecer la pasión del viajero en estos sitios con el principio de conservar los recursos naturales.

\section{MATERIALES Y MÉTODOS}

El Refugio de Vida Silvestre Río Escalante - Chacocente se encuentra ubicado entre el municipio de Santa Teresa, departamento de Carazo y el municipio de Tola, departamento de Rivas, entre las coordenadas $11^{\circ} 30^{\prime} 33.0^{\prime \prime} \mathrm{N}, 11^{\circ} 35^{\prime} 28.5^{\prime \prime} \mathrm{O}$ y $86^{\circ} 08^{\prime} 33.7^{\prime} \mathrm{N}, 86^{\circ} 14^{\prime} 43.1$ 'O; tiene una extensión de 4 645.4 ha $\left(46.45 \mathrm{~km}^{2}\right)$, el clima predominante es típico de las zonas cálidas secas y la temperatura oscila entre $26^{\circ} \mathrm{C}$ y 29 ${ }^{\circ} \mathrm{C}$, con una precipitación media anual entre 900 y $1200 \mathrm{~mm}$, la época lluviosa es de mayo a octubre (Buitrago, 2013).

La investigación es de tipo descriptiva no experimental con análisis cualitativo; la metodología aplicada es tomada y modificada de la guía para el diseño y operación de senderos interpretativos propuesta por la Secretaría de Turismo de México (SECTUR MX, 2004) para un sendero en forma lineal-circuito, con la modalidad turística responsable conocida como ecoturismo, definida por Ceballos-Lascuráin (1998). Basado en las características del sitio (ecosistema tropical seco y de playa), se consideró la identificación de la flora y avifauna silvestre representativa; un guardabosque asignado colaboró en la identificación dentrológica y para el inventario de la avifauna y especies marinas costeras, se tomaron fotografías y en colaboración del Lic. Miguel Garmendia de la UNA, se obtuvo sus nombres científicos. De esta manera, el estudio se desarrolló en tres fases.

Fase 1. Organización, revisión, coordinación y planificación de la investigación. a) Contactos iniciales con tres funcionarios de la dirección de patrimonio cultural del MARENA Central (Ing. Carlos Mejía, Lic. Rene Castellón - Dirección de Biodiversidad y Dilcia Solano - Dirección de SINAP) para presentar la propuesta integral del proyecto Manejo Forestal diversificado con enfoque comunitario para el RVS Rio Escalante - Chacocente (2016), y dentro del cual, se incluyó la presente investigación; esto conllevó gestiones de solicitud de certificación de permiso de entrada al área. b) Compilación y revisión de información secundaria referente al tema (metodologías acordes con los objetivos planteados y de tipos de inventarios de potenciales y de flora y fauna a realizar). c) Planificación de visitas de reconocimiento al sitio y senderos y de fechas y días de campo para recabar la información requerida, coordinado con la administración del refugio.

Fase 2. Toma de datos de campo, observaciones in situ, geo referenciación de puntos y senderos, inventario de especies de flora arborescente, avifauna silvestre $y$ potenciales naturales. a) Aplicación de formatos varios: de inventario de potenciales naturales, de especies arbóreas nativas, de avifauna silvestre y de tipo de formaciones forestales presentes en las áreas de los dos senderos cercanos a las instalaciones del Refugio, y de especies de playa en las zonas de afloramiento costero. b) Georreferenciación de recorridos y de estaciones interpretativas identificadas dentro de cada sendero encontrado y de distancia entre ellas. c) Aplicación de la técnica de observación directa para la descripción cualitativa de los potenciales naturales $\mathrm{y}$ de las estaciones en un plano horizontal y vertical (que hay, cómo esta, qué la rodea, que actividades ecoturísticas se podría realizar en cada una según tipo de usuario, qué contenido se podría abordar durante el guiado de turistas y cuánto tiempo de estadía se demanda en cada una) durante los recorridos por los senderos, esto se llevó a cabo con el acompañamiento de dos guarda parques del área protegida y 
con la implementación del formato de inventario de atractivos prediseñado según la metodología de la Secretaría de Turismo de México (SECTUR MX, 2004). d) Descripción de posibles actividades ecoturísticas a realizar en el refugio, que fueron presentadas a tres guarda bosques del refugio y concretadas con información de las entrevistas no estructuradas que se les aplicaron a ellos al momento de realización de los recorridos. e) Determinación de la flora representativa utilizando dos metodologías: (1) reconocimiento en campo con el acompañamiento de un guardabosque del refugio especializado en flora nativa y (2) con la revisión documental y aportes brindados por Benito Quezada, docente de la facultad de Recursos Naturales y del Ambiente (FARENA) de la Universidad Nacional Agraria (UNA). f) Determinación de la fauna silvestre: (1) revisión de trabajos realizados en el área de estudio y (2) reconocimiento en campo.

Fase 3. Análisis y descripción de la información recabada. a) Elaboración de la propuesta de recorridos por dos senderos ecoturístico tipo lineal - circuito con sus estaciones interpretativas. Este mapa temático se logró editar con el uso del programa de cómputo ArcGIS 10.5 (ESRI, 2016) e imágenes LANDSAT 8. b) Diseño de cinco rótulos interpretativos; estos dibujos propuestos plasman los cuatro criterios que establece como marco las Normas Jurídicas de Nicaragua (2018), y se procedió a digitalizarlos utilizando programas gráficos como Microsoft Paint, Paint Shop Pro y Microsoft PowerPoint. c) Elaboración del material didáctico; se diseñó una cartilla con el programa Adobe InDesign y para los dibujos el Microsoft Paint y Paint Shop Pro; con la información recabada del estudio se divulga el quehacer del refugio, se promueve la educación ambiental y se concientiza a la población en temas de importancia ambiental, su contenido incluye galería de fotos del refugio, de juegos interactivos y de temarios y conceptos varios relacionados con la razón de ser del refugio; su boceto final fue consensuado con la administración del refugio y con el director de patrimonio cultural Lic. MSc. Rene Castellón.

\section{RESULTADOS Y DISCUSIÓN \\ Inventario de potenciales naturales del RVS}

Potenciales naturales. Se identificaron 11 potenciales naturales ubicados en los Senderos Cola Chata y Tamarindo, hasta la zona marino-costera; para cada uno se proponen actividades ecoturísticas que pueden realizar los diversos grupos de usuarios (nacionales, extranjeros, investigadores, estudiantes de los diversos grados académicos y público en general) que visiten la zona oeste del refugio (Cuadro 1), pero estas, se elaboraron tomando en cuenta el plan de manejo vigente y las orientaciones de la administración del refugio, es por ello que están dirigidas a la promoción de la recreación con fines conservacionista y de la educación ambiental.

Cuadro 1. Potenciales naturales, actividades ecoturísticas y potenciales usuarios del RVS Río Escalante - Chacocente, 2017

\begin{tabular}{|c|c|c|}
\hline Potenciales naturales & Propuestas de actividades ecoturísticas & Potenciales usuario \\
\hline $\begin{array}{l}\text { 1. Playa de anidación de } \\
\text { tortugas }\end{array}$ & $\begin{array}{l}\text { Interpretación del medio natural, } \\
\text { educación ambiental, investigación } \\
\text { científica }\end{array}$ & $\begin{array}{l}\text { Visitantes nacionales, extranjeros e } \\
\text { investigadores }\end{array}$ \\
\hline 2. Especies arbóreas nativas & $\begin{array}{l}\text { Descripción morfológica de la flora } \\
\text { nativa, de aspectos sobresalientes, } \\
\text { tipos de flores y frutos, historia natural } \\
\text { e importancia económica y ecológica }\end{array}$ & $\begin{array}{l}\text { Visitantes nacionales, extranjeros, } \\
\text { investigadores y estudiantes de } \\
\text { diversos grados académicos }\end{array}$ \\
\hline 3. Fauna silvestre & $\begin{array}{l}\text { Avistamiento de aves, de especies } \\
\text { marino costera, de playa y zonas } \\
\text { rocosas, su belleza, importancia } \\
\text { ecológica, } \\
\text { carismáticas }\end{array}$ & $\begin{array}{l}\text { Visitantes nacionales, extranjeros, } \\
\text { investigadores y estudiantes de } \\
\text { diversos grados académicos }\end{array}$ \\
\hline $\begin{array}{l}\text { 4. Arribada de tortugas } \\
\text { marinas }\end{array}$ & $\begin{array}{l}\text { Interpretación del medio natural, } \\
\text { observación de tortugas marinas, } \\
\text { aspectos morfológicos, veda y } \\
\text { reproducción, educación ambiental, }\end{array}$ & $\begin{array}{l}\text { Visitantes nacionales, extranjeros, } \\
\text { investigadores y estudiantes de } \\
\text { diversos grados académicos }\end{array}$ \\
\hline $\begin{array}{l}\text { 5. Vistas panorámicas o } \\
\text { paisajismo y belleza escénica } \\
6 \text {. Ecosistemas del Bosque } \\
\text { tropical seco } \\
\text { 7. Manglares } \\
\text { 8. Transición playa - bosque } \\
\text { 9. Afloramientos costero- } \\
\text { rocosos } \\
\text { 10. Marino }\end{array}$ & $\begin{array}{l}\text { Caminata por senderos interpretativos, } \\
\text { fotografías de paisajes y naturaleza } \\
\text { Establecimiento de campamentos, } \\
\text { interpretación del medio natural, } \\
\text { fotografías de paisajes y naturaleza, } \\
\text { educación ambiental. }\end{array}$ & $\begin{array}{l}\text { Visitantes nacionales y extranjeros, } \\
\text { investigadores } \\
\text { Visitantes nacionales, extranjeros, } \\
\text { investigadores y estudiantes de } \\
\text { diversos grados académicos }\end{array}$ \\
\hline 11. Playa & Balneario y surf & Visitantes nacionales y extranjeros \\
\hline
\end{tabular}


Estos potenciales considerados atractivos turísticos por los administradores del área (Normas Jurídicas de Nicaragua, 2018), presentan diversas oportunidades recreativas y prácticas para ser disfrutadas durante los recorridos, ya que incluyen desde la observación de diversos paisajes, de avistamiento de aves, diversidad de ecosistemas y de coloridas formas de vida vegetal en tiempos diurnos y nocturnos y que están en dependencia de la época del año en que sean visitado. Para poder acceder al disfrute de potenciales y/o realización de actividades, se debe contar con un permiso de entrada que emite el MARENA central y en la administración de refugio.

Diversidad biológica. Con el inventario de la flora representativa (Cuadro 2) y con la revisión bibliográfica y los avistamientos de campo sobre fauna silvestre autóctona (Cuadro 3) realizado en los dos senderos cercanos al refugio, se pudo identificar 24 especies arbóreas nativa; y consensuar una lista de 27 especies de fauna silvestre vertebrada e invertebrada, sin incluir a las tortugas marinas; especies que en general pueden ser atractivas para los visitantes, en especial por ser especies carismáticas que podrían impresionar a visitantes nacionales o extranjeros y despertar el espíritu investigador de aquellos interesados en verlas en su ambiente natural.

Con los potenciales naturales identificados en un área protegida, se pueden destacar la importancia del medio natural en donde el ser humano y la naturaleza pueden interactuar, representando para el visitante un alto valor ambiental y social, que le permite establecer actividades ecoturísticas, como lo establece Mayorga y Carrión (2017). Así mismo, la Secretaría de Turismo de México (SECTUR MX, 2004) considera el ecoturismo como una forma de turismo alternativo; y para Ceballos-Lascuráin, (1998), consiste en una forma de visitar o viajar a áreas naturales con el fin de disfrutar, apreciar y estudiar los atractivos naturales (paisaje, flora y fauna silvestres) de dichas áreas, así como cualquier manifestación cultural (del presente y del pasado) que pueda encontrarse allí, a través de un proceso que promueve la conservación, que tiene bajo impacto ambiental y cultural, y propicia una participación activa de las poblaciones locales, socio económicamente beneficiosa para éstas.

\section{Diseño de senderos interpretativos ecoturístico-educativo Sendero Lagarto Cola Chata - El Tamarindo del Refugio de Vida Silvestre Río Escalante - Chacocente. Cercano} a la Estación Biológica del refugio, se encontraron dos senderos, uno llamado Lagarto Cola Chata debido a la presencia de una especie muy particular de reptil llamada Lagarto Cola Chata (Ctenosaura quinquecarinata) que habita en un árbol de Nacascolo en la entrada al sendereo, con una extensión de $400 \mathrm{~m}$ y tiene dirección Norte Sur; y otro El Tamarindo con una extensión de $600 \mathrm{~m}$.
Cuadro 2. Fauna silvestre por nombre común y científico de la RVS, 2017

\begin{tabular}{lll}
\hline Grupo & Nombre común & Nombre científico \\
\hline Vertebrado & Anemonas & Actinernidae Halcuriidae \\
Vertebrado & Ardilla chiza & Sciurus variegatoides \\
Invertebrado & Babosa marina & Tridachiella diomedea \\
Invertebrado & Barnacla de Percebe común & Chthamalus stellatus \\
Vertebrado & Boa & Boa imperator \\
Invertebrado & Caracol con puntas naranjas & Jenneria pustulata \\
Vertebrado & Conejo & Sylvilagus sp. \\
Invertebrado & Erizo barbiado & Diadema mexicanum \\
Invertebrado & Erizo de lápiz de pizarra & Eucidaris thouarsii \\
Invertebrado & Estrella de mar & Phantaria unifascialis \\
Invertebrado & Estrella frágil negra & Ophicomina nigra \\
Vertebrado & Garrobo & Ctenosaura similis \\
Vertebrado & Garrobo cola chata & Ctenosaura quinquecarinata \\
Vertebrado & Gavilán gris & Asturina nitida \\
Vertebrado & Guardabarranco & Eumomota superciliosa \\
Vertebrado & Guatusa & Dasyprocta punctata \\
Vertebrado & Iguana & Iguana iguana \\
Vertebrado & Lagartija & Aspidodoscelis deppil \\
Vertebrado & Mano de piedra & Gymnopis multiplicata \\
Vertebrado & Mica & Spilotes pullatus \\
Vertebrado & Mono araña & Ateles geoffroyi \\
Vertebrado & Mono aullador & Alouatta palliata \\
Vertebrado & Pájaro carpintero & Melanerpes hoffmannii \\
Vertebrado & Saltarín Toledo & Chiroxiphia linearis \\
Vertebrado & Serpiente cascabel & Crotallus durissus \\
Vertebrado & Urraca copetona & Calocitta formosa \\
Vertebrado & Zanate negro & Quiscalus mexicanus \\
Vertebrado & Zorro pelón & Didelphis marsupialis \\
\hline & &
\end{tabular}

Cuadro 3. Flora representativa por nombre común y científico de la RVS, 2017

\begin{tabular}{cc}
\hline Nombre común & Nombre científico \\
\hline Barbasco & Bonellia nervosa \\
Botoncillo & Conocarpus erectus \\
Brasil & Haematoxylum brasiletto \\
Cactus & Acanthocereus tetragonus \\
Cachito & Stemmadenia spp \\
Caoba & Swietenia humilis zucc \\
Cornizuelo & Acacia costaricensis \\
Cortez & Tabebuia ochraceae ssp neochysantha \\
Chaperno & Albizia adinocephala \\
Chiquirín & Myrospermum frutescens \\
Chocoyito & Dyospirus nicaraguensis \\
Espino de playa & Pithecellobium dulce \\
Guácimo de molenillo & Luehea candida \\
Guácimo de terneo & Guazuma ulmifolia \\
Guanacaste blanco & Albizzia caribea \\
Huevo de chancho & Stemmadenia obovata \\
Jiñocuabo & Bursera simaruba \\
Madroño & Calycophyllum candidissimum \\
Melero & Thounidium decandrum \\
Nacascolo & Caesalpinia coriaria (Jacq.) Willd \\
Nanciguiste & Zizyphus guatemalensisi \\
Palancón & Sapranthus nicaraguensis \\
Piñuelas & Bromelia pingui \\
Talalate & Gyrocarpus americanus \\
Tostadillo & Allospryllum occidental \\
\hline &
\end{tabular}


Estaciones interpretativas. En este sentido y debido a la importancia ecológica y ambiental del refugio, se consideró destacar sus potenciales naturales desde la perspectiva de estaciones interpretativas, con la distancia entre ellas, en conectividad con los senderos, tiempo y tipo de recorrido; se identificaron nueve estaciones interpretativas para un solo recorrido propuesto, al cual se le llamó sendero Lagarto Cola Chata - Tamarindo con una extensión total $2.5 \mathrm{~km}$; recorrido lineal abierto corto (si solo se realiza parte del recorrido) y circuito que inicia y termina en la Estación Bilógica, y se puede hacer en aproximadamente dos horas; es de tipo guiado y autoguiado (Figura 1). En el Cuadro 4, se describe por orden cada estación.

Figura 1. Ruta del sendero ecoturístico-educativos Lagarto Cola Chata-El Tamarindo, del Refugio de Vida Silvestre Río Escalante Chacocente, Carazo-Rivas, 2018

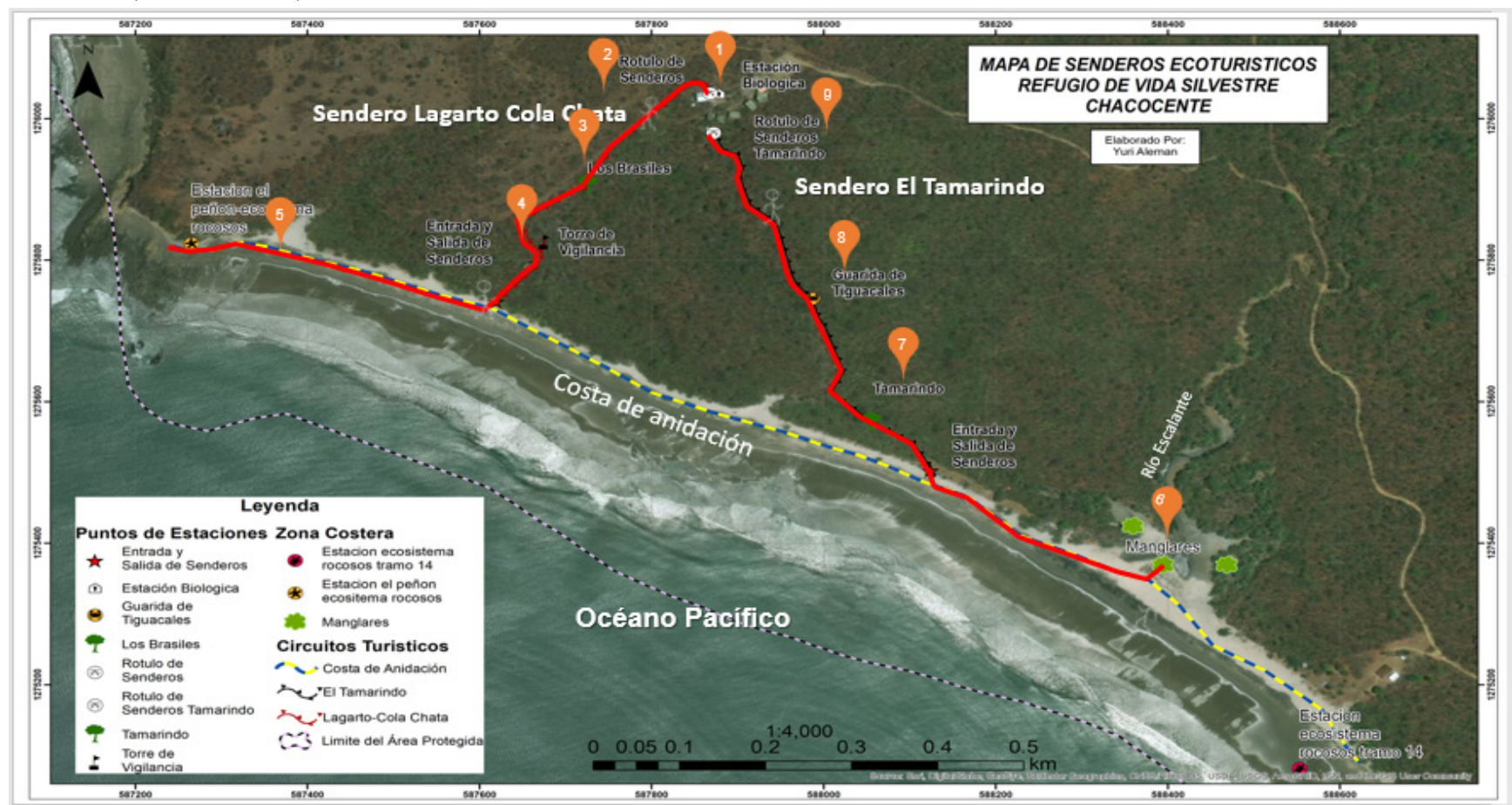

Cuadro 4. Estaciones interpretativas establecidas en el Sendero ecoturístico-educativos Lagarto Cola Chata - Tamarindo del Refugio de Vida Silvestre Río Escalante - Chacocente, 2017

\begin{tabular}{|c|c|c|}
\hline & $\begin{array}{c}\text { Estaciones } \\
\text { interpretativas }\end{array}$ & Descripción general \\
\hline 1 & Estación biológica & $\begin{array}{l}\text { Consiste en una serie de cabañas que alberga al personal del refugio, personal del ejército } \\
\text { y a los visitantes. En esta se encuentra la recepción y dirección del área protegida y es aquí } \\
\text { donde el visitante llega a registrarse antes de dirigirse a cualquier área dentro del refugio. }\end{array}$ \\
\hline 2 & $\begin{array}{l}\text { Parada } \\
\text { informativa }\end{array}$ & $\begin{array}{l}\text { Rótulo interpretativo, ubicado en el sendero Lagarto Cola Chata a } 10 \mathrm{~m} \text { de la Estación } \\
\text { Biológica y se puede llegar en un tiempo no mayor de un minuto. Si el recorrido es } \\
\text { guiado, el guarda de área natural explica y orienta la ruta que se llevará durante el } \\
\text { recorrido por el sendero en un tiempo aproximado de } 4 \text { minutos. }\end{array}$ \\
\hline 3 & Los Brasiles & $\begin{array}{l}\text { Se encuentra a } 144 \text { m de la parada o Rótulo interpretativo y este recorrido consiste en una } \\
\text { caminata en forma de zic zac aproximada de siete minutos. Durante la caminata, se puede } \\
\text { observar flora nativa del bosque seco del refugio, entre las cuales sobresalen: Acacia } \\
\text { costaricensis (cornizuelo), Pithecellobium dulce (espino negro), Ajacquinia aurantioca } \\
\text { (barbasco), Stemmadenia spp (cachito). }\end{array}$ \\
\hline 4 & $\begin{array}{l}\text { Casar múltiple } \\
\text { (Torre de } \\
\text { observación) }\end{array}$ & $\begin{array}{l}\text { Desde la belleza en biodiversidad que ofrece la estación Los Brasiles, siguiendo el sendero } \\
\text { unos } 220 \mathrm{~m} \text { ( } 10 \text { minutos de caminata) hacia el Sur se encuentra la Casa múltiple. Esta casa } \\
\text { es ocupada exclusivamente por los guardas de áreas protegidas cuando se dan las arribadas } \\
\text { masivas de tortugas marinas, sirve para vigilancia. }\end{array}$ \\
\hline
\end{tabular}


Cuadro 4. Continuación...

\begin{tabular}{|c|c|c|}
\hline 5 & El Peñón & $\begin{array}{l}\text { Este se encuentra saliendo a la playa y tomando rumbo Oeste a unos } 800 \mathrm{~m} \text { (10 minutos } \\
\text { caminando) la estación. Esta estación representa un ecosistema rocoso rico en diversidad } \\
\text { de vida animal y vegetal, que se desarrolla entre los repetidos cambios de marea. Es fácil } \\
\text { observar epifauna marina, organismos vivos que habitan en las rocas especies como: } \\
\text { bálanos (Chthamalus stellatus), Caracol putas naranjas (Jenneria pustulata), Erizo de lápiz } \\
\text { de pizarra (Eucidaris thouarsii), babosa marina (Tridachiella diomedea), Estrella de mar } \\
\text { (Phantaria unifascialis), Erizo barbiado (Diadema mexicanum), entre otros. }\end{array}$ \\
\hline 6 & E1 Manglar & $\begin{array}{l}\text { La estación El Manglar (opcional) se encuentra a } 900 \mathrm{~m} \text { ( } 20 \text { minutos caminando) desde El } \\
\text { Peñón, rumbo Este. Es uno de los atractivos naturales que conforma uno de los } \\
\text { ecosistemas más importante de nuestro país, como lo es el manglar y la playa de } \\
\text { anidación. Algunas especies en particular podrían ser abundantes y muy atractivas como } \\
\text { por ejemplo el cangrejo de mangle (Aratus pisonii), los camarones (Litopenaeus sp.), las } \\
\text { conchas negras (Anadara tuberculosa), entre otras especies, incluyendo una exuberante } \\
\text { diversidad de peces. Algunas especies de flora representativas de este ecosistema son el } \\
\text { mangle (Rhizophora mangle), el palo de sal (Avicennia bicolor) y palo de sal (Germinans } \\
\text { sp). }\end{array}$ \\
\hline 7 & El Tamarindo & $\begin{array}{l}\text { Caminando sobre la playa hacia el Este, a unos } 10 \text { minutos }(300 \mathrm{~m}) \text { del Peñón, se llega a la } \\
\text { entrada del sendero El Tamarindo. En esta estación encontramos un descanso ideal bajo la } \\
\text { sombra del frondoso árbol de Tamarindo (Tamarindus indica L.), una especie con } \\
\text { importancia económica pues sus frutos son utilizados para elaborar refrescos y } \\
\text { mermeladas. En esta estación habita un tipo de cangrejo en particular que es comúnmente } \\
\text { conocido como cangrejo de Halloween o piñuelero (Gecarcinus quadratus), una especie } \\
\text { muy vistosa por sus colores negros y naranja. También, esta puede visitarse desde la } \\
\text { estación biológica tomando la dirección de Norte a Sur con una extensión de } 600 \text { metros } \\
\text { hacia la playa. Si es así, solamente podrán considerarse las estaciones Parada o Rótulo de } \\
\text { interpretación, La Guarida de los Tiguacales, El Tamarindo y El Manglar. }\end{array}$ \\
\hline 8 & $\begin{array}{l}\text { La guarida de los } \\
\text { Tiguacales }\end{array}$ & $\begin{array}{l}\text { A } 250 \text { m ( } 7 \text { minutos de camino sobre la playa) del Rótulo interpretativo Sendero El } \\
\text { Tamarindo, se encuentra La guarida de los Tiguacales. El nombre se deriva de la presencia } \\
\text { de una especie de cangrejos que se caracteriza por tener el cefalotórax de color azul- } \\
\text { celeste llamado tiguacal (Cardisoma crassum). Además del Tiguacal, se pude observar } \\
\text { una gran variedad de especies de flora típicas del bosque seco tropical, entre ellas están las } \\
\text { piñuelas (Bromelia pingui), los cactus (Acanthocereus tetragonus), el huevo de chancho } \\
\text { (Stemmadenia obovata), el palo de maya (Mimosa platucarpa), entre otras. }\end{array}$ \\
\hline 9 & $\begin{array}{l}\text { Parada } \\
\text { informativa }\end{array}$ & $\begin{array}{l}\text { Rótulo interpretativo Sendero El Tamarindo. Desde la entrada Este se encuentra a una } \\
\text { distancia de } 50 \mathrm{~m} \text { y por la entrada Sur de la Estación Biológica a } 4 \text { minutos. En esta parada } \\
\text { temática el guardabosque podría brindar información acerca del sendero, de las } \\
\text { actividades que se pueden hacer en él y de los potenciales naturales que se pueden } \\
\text { observar al momento de la caminata. }\end{array}$ \\
\hline
\end{tabular}

Propuesta de rótulos interpretativos. Se encontraron diversos rótulos caídos y sin una clara información, es por lo que se proponen dos rótulos, uno por sendero, para concretar el recorrido total lineal - circuito del sendero Lagarto Cola Chata - Tamarindo. Estos ofrecen información para el tipo de visita auto guiada y señalizaciones de los atractivos naturales que se pueden apreciar en los senderos (Figura 2 y 3 ).

En la investigación realizada por Bazurto (2018) se menciona que un sendero interpretativo es considerado como una actividad de turismo alternativo que va dentro del segmento de ecoturismo, donde básicamente, el visitante recorre a pie o en transporte no motorizado, un camino o sendero predefinido y equipado con letreros de información ambiental. Por otro lado, Pellegrini (2009), expresa que la interpretación ambiental se vale de medios para llegar a sus destinatarios, los cuales deben incluir instalaciones, actividades y materiales.

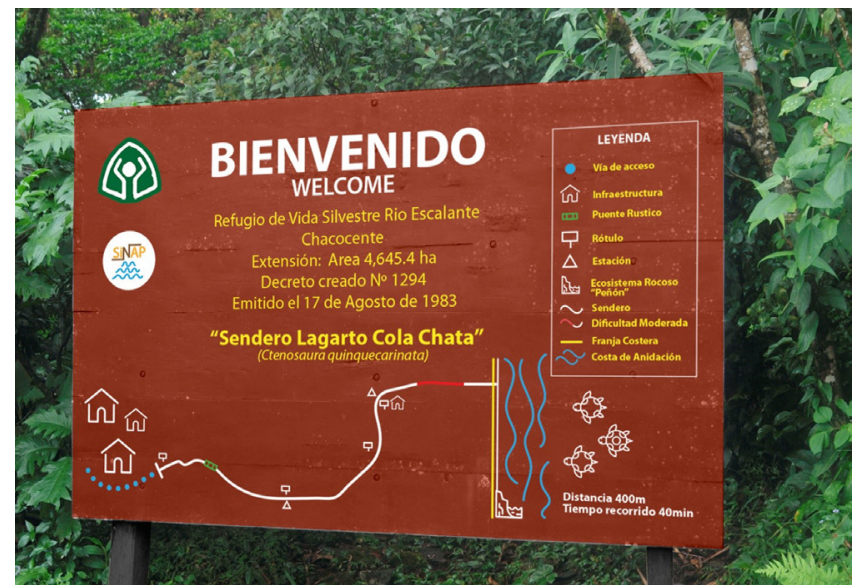

Figura 2. Propuesta de rótulo interpretativo para el sendero Lagarto Cola Chata, 2018. 
Estos medios refieren un itinerario o recorrido preestablecido por lugares con determinadas características, en el que se establece una secuencia ordenada de paradas con diversos recursos (elementos o procesos observables y atractivos) que, en conjunto, presentan un mensaje o tema relacionado con el conocimiento, la valoración y la conservación del espacio. Complementado por Ramírez (2009) debe usarse como una herramienta educativa para ser aplicada en áreas naturales o espacios protegidos donde se llevan a cabo actividades educativas que buscan la integración de la sociedad civil a los procesos de conservación de algún área en particular. Por lo tanto, se considera de gran importancia aportar con la presente.

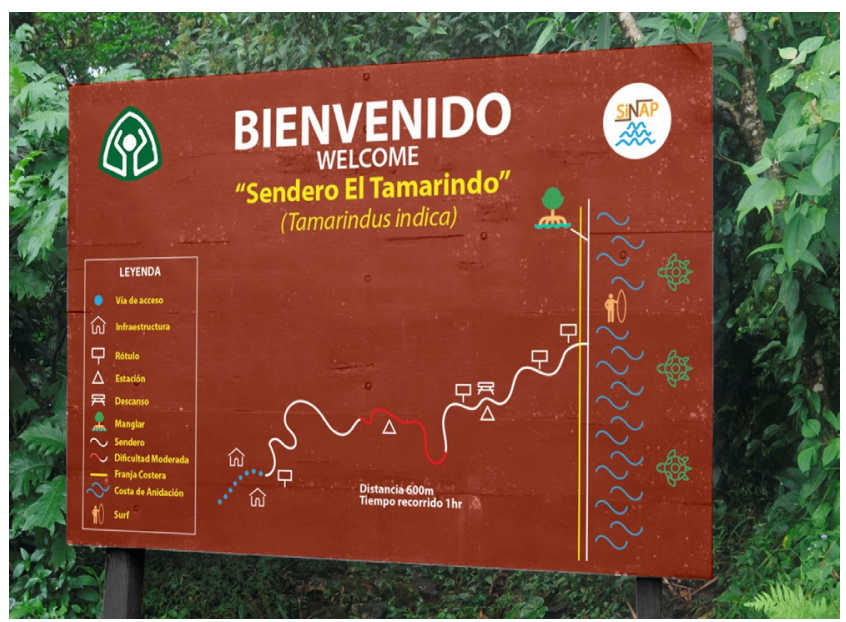

Figura 3. Propuesta de rótulo interpretativo para el sendero El Tamarindo, 2018.

\section{Propuesta de cartilla "Haciendo Educación Ambiental"}

Diseño del material didáctico. Este material didáctico se diseñó con la finalidad de brindar información ambiental, ecológica, y del refugio a los visitantes y comunitarios. Además, contiene pasatiempos y dossier fotográfico que provee de atractivo visual a los lectores.

Atributos de la cartilla. Cuenta con cuatro atributos importantes que son: (1) Secuencia lógica (Estructurado en orden lógico), (2) Articulación espacio - tiempo (Comunicación visual con una secuencia temporal de lo que se puede observar y los elementos que facilitan su lectura), (3) Lenguaje claro y popular (Abierto para un público local que capte el mensaje desde diversos enfoques de los lectores), y (4) Elementos gráficos (que incluyen ilustraciones, imágenes, tipografía y diagramación y juegos interactivos de manualidades y actividades mentales).

Contenido de la cartilla. Está ordenado en tres secciones: (1) Las iniciales, que incluyen los agradecimientos, el prólogo y la introducción; (2) Del cuerpo, que abarca la base conceptual de Educación Ambienta, del Ecoturismo, potenciales naturales del refugio, actividades ecoturísticas, juegos y pasatiempos, que en general, tienen énfasis en la conservación, conocimiento de la naturaleza y la biodiversidad (especies de la vida terrestre o marina) del refugio; y (3) De finalización, que son la bibliografía y el glosario.

$\mathrm{Al}$ relacionar este estudio con el de Bazurto (2018), Pellegrini (2009) y Ramírez (2009), se puede constatar la importancia de enfocar los potenciales o atractivos de un sitio con el ecoturismo y asegurar el disfrute de una serie de placeres a través de actividades in situ que se plantea ofertar a los visitantes a través de una ruta diseñada de circuito con un inicio (entrada) y un fin (salida), plasmada en una imagen, plano o croquis con las estaciones pre definidas entre sitios $\mathrm{y}$ tener como lenguaje lo comunicacional (acompañamiento técnico) tal y como lo recomienda Castillo (2017) y la Secretaría de Turismo de México (SECTUR MX, 2004); incluso de un material didáctico complementario, como en este caso, una Cartilla de educación ambiental, ecológica y biológica, y práctica con lenguaje sencillo para todo tipo de usuario.

\section{CONCLUSIONES}

Se determinaron 11 potenciales naturales en los senderos, asociados con diversas actividades recreativas ecoturísticas como la interpretación de medios naturales, observación de flora y fauna, investigación científica, caminata por los senderos, fotografía de elementos naturales y paisajes, surf y otros.

Los potenciales o atractivos naturales identificados sirvieron de base para rehabilitar la ruta del sendero Lagarto Cola Chata y para conectarlo con la ruta del sendero El Tamarindo. El sendero Lagarto Cola Chata-El Tamarindo consta de nueve estaciones: estación biológica, rótulo interpretativo 1, Los Brasiles, Casa Múltiple - Torre de observación, El Peñón, El Manglar, El Tamarindo, Guarida de Los Tiguacales y rótulo interpretativo 2 .

Fue posible el diseño de un mapa o croquis de la Ruta del sendero ecoturístico-educativos Lagarto Cola ChataEl Tamarindo, del Refugio de Vida Silvestre Río Escalante - Chacocente sendero, de siete elementos interpretativos (rótulos), dos de ellos describen la ruta de cada sendero, el resto de los rótulos están destinado a brindar direcciones e información de elementos particulares de las estaciones y del área protegida.

Como material didáctico se diseñó una cartilla llamada "Haciendo Educación Ambiental" la cual fue escrita siguiendo atributos para su elaboración y concentra información arreglada en cinco secciones. 


\section{REFERENCIAS BIBLIOGRÁFICAS}

Bazurto Cevallos, K. E. (2018). Diseño de un sendero interpretativo en el bosque seco tropical, sitio - El Retiro, como aporte al desarrollo ecoturistico del Cantón JAMA. [Tesis de pregrado, Universidad Laica Eloy Alfaro de Manabi]. Repositorio Institucional ULEAM. https://repositorio.uleam.edu.ec/bitstream/123456789/1110/1/ULEAM-HT-0012.pdf

Buitrago Vaninni, F. (2013). Areas protegidas de Nicaragua: primer tomo, Región del pacifico. FUNDENIC SOS.

Castillo Conde, C. M. (2017). Importancia del desarrollo turístico del circuito vivencial amazonas y su influencia en la economía de la región - 2017. [Tesis de pregrado, Universidad de San Martín de Porres]. Repositorio Académico USMP. http://repositorio.usmp. edu.pe/bitstream/handle/20.500.12727/3399/castillo_ccm.pdf?sequence=3\&isAllowed=y

Ceballos-Lascuráin, H. (1998). Ecoturismo en Centro América. Reporte Técnico para OMT/UNDP.

Cifuentes, M. A., Mezquita, C., Méndez, J. (1999). Capacidad de carga turística de las áreas de uso público del Monumento Nacional Guayabo, Costa Rica. WWF Centroamérica. http://awsassets.panda.org/downloads/wwfca_guayabo.pdf

Ejzman, M. A. (2000). Planificación ecoturística y capacidad de carga. https://www.gochile.cl/spa/Guide/ChileSeminarioEcoturismo/ Ponencias

Mayorga Arosteguí, J. E., y Carrión D. E. (2017). Diseño de un sendero ecoturístico para la finca de conservación Ecológica "Reserva Hidrica Forestal-ADP”, municipio San José de los Remates, Boaco, Nicaragua. [Tesis de pregrado, Universidad Nacional Agraria]. Repositorio Institucional UNA. http://repositorio.una.edu.ni/3560/1/tnp01m473.pdf

Ministerio del Ambiente y los Recursos Naturales, Nicaragua. (2008). Plan de manejo de refugio de vida silvestre rio Chacocente. http://www.bvsde.org.ni/Web_textos/MARENA/CDInteractivo0002/imagenes/chacocente.pdf

Normas Jurídicas de Nicaragua. (2018). Normas para la rotulación, señalización ambiental y turística en las áreas protegidas del sistema nacional de áreas protegidas SINAP. http://legislacion.asamblea.gob.ni/normaweb.nsf/(\$All)/ D416FF97C6A9A9F5062582FA0049A631?OpenDocument

Olivares López, D; Obiol Menero, E. M. (2005). El turismo en Nicaragua. Un análisis territorial contemporáneo. ERIAS, 67, $209-218$. https://dialnet.unirioja.es/descarga/articulo/1393273.pdf

Pellegrini, N. (2009). Sendero de interpretación ambiental en el Bosque de la Universidad Simón Bolívar. Sapiens. Revista Universitaria de Investigación, 10(2), 47-67. https:/www.redalyc.org/articulo.oa?id=41021266003

Ramírez., A. (2009). Sendero interpretativo de Agroecoturismo entre los cerros orientales de Bogotá y el Parque Nacional Natural Chingaza. Asociación CEAMOS.

Secretaría de Turismo, México. (2004). Guía para el diseño y operación de senderos interpretativos. http://www.jjcano.com/wpcontent/ uploads/2011/01/11_conceptualizacion_turismo_alternativo1.pdf 